\title{
Structure factors of harmonic and anharmonic Fibonacci chains by molecular dynamics simulations
}

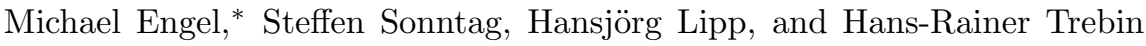 \\ Institut für Theoretische und Angewandte Physik, \\ Universität Stuttgart, Pfaffenwaldring 57, 70550 Stuttgart, Germany
}

\begin{abstract}
The dynamics of quasicrystals is characterized by the existence of phason excitations in addition to the usual phonon modes. In order to investigate their interplay on an elementary level we resort to various one-dimensional model systems. The main observables are the static, the incoherent, and the coherent structure factor, which are extracted from molecular dynamics simulations. For the validation of the algorithms, results for the harmonic periodic chain are presented. We then study the Fibonacci chain with harmonic and anharmonic interaction potentials. In the dynamic Fibonacci chain neighboring atoms interact by double-well potentials allowing for phason flips. The difference between the structure factors of the dynamic and the harmonic Fibonacci chain lies in the temperature dependence of the phonon line width. If a bias is introduced in the well depth, dispersionless optic phonon bands split off.
\end{abstract}

PACS numbers: 63.20.Ry, 61.44.Br, 02.70.Ns Keywords: Phonons; Phason flip; Quasicrystal.

\section{INTRODUCTION}

\section{A. Motivation}

Quasicrystals are long-range ordered materials lacking translational symmetry ${ }^{1}$. Their diffraction patterns exhibit a dense set of sharp Bragg reflections, that can be indexed by an integer linear combination of a finite number of basis vectors which is larger than the dimension of space. As a consequence the atomic configuration of quasicrystals is described with reference to a higherdimensional analog of a periodic lattice. Elementary dynamic excitations within this 'hyperspace' description are phonons ${ }^{2}$ and phasons ${ }^{3}$.

Phasons involve rearrangements of the structure by atomic jumps over short distances, denoted 'phason flips'. They are connected with many physical properties of quasicrystals as for example elastic deformations 4 , dislocations $\sqrt{5 / 6}$, diffusion ${ }^{7 / 8}$, and phase transformations ${ }^{9}$. Recently, indications for phason flips ${ }^{1011}$ have been observed by in situ transmission electron microscopy. A coherent set of phason flips may form a static phason field, e.g. during a phase transformation or in the neighborhood of a dislocation 12 .

By investigating the dynamics of quasicrystals one can find out the influence of the quasiperiodicity on the phonon spectrum ${ }^{2}$ and one may gain a deeper understanding of phason flips ${ }^{13}$. Both points can be studied in x-ray or neutron diffraction experiments by measuring the response of the system in frequency $(\omega)$ and momentum $(q)$ space. Depending on the experimental setup, different functions can be obtained from the scattering experiments: (1) The static structure factor $S(q)$ is the usual - not energy resolved - diffraction image, measured with either x-rays or neutrons. It is used for the determination of the atomic structure. (2) The coherent structure factor ${ }^{14} S(q, \omega)$ is studied via coherent inelastic neutron scattering $\sqrt{15}$ or alternatively via inelastic x-ray scattering 16 . It allows the determination of the phonon dispersion relations. The experiments on icosahedral quasicrystals show well defined acoustic phonon modes at small wave-vectors 17 and dispersionless broad

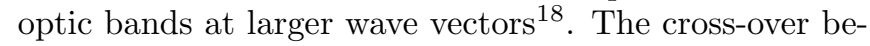
tween the two regions is very sharp. (3) The incoherent structure factor ${ }^{14} S_{\mathrm{i}}(q, \omega)$ can be measured in quasielastic neutron scattering. Neutrons are exclusively used here, due to the necessity of a high energy resolution. The technique also allows the investigation of phason flips. In a series of experiments Coddens et al!13]19/20|21 have found an anomalous $q$-dependence of the quasielastic signal in icosahedral quasicrystals. They interpreted it as correlated simultaneous jumps of several atoms.21

Up to now various calculations of the coherent structure factor of quasiperiodic model systems have been published, see Ref. 2. Amongst them are the perfect onedimensional Fibonacci chain 22 , by static phason fields disordered Fibonacci chains ${ }^{23}$, and three-dimensional tilings 24 . In these studies the dynamical matrix is diagonalized, which is a purely analytic method and yields the phonon dispersion relations only. The results are highly structured excitation spectra with a hierarchical system of gaps 22 . The influence of anharmonicities, however, especially the dynamics of phason flips has not been taken into account.

This 'missing link' markes the starting point of our study. Here we present calculations of the structure factors of special one-dimensional quasiperiodic model systems by use of molecular dynamics (MD) simulations with either harmonic potentials or potentials that allow for phason flips. Although structure factors play such a central role in the dynamics of solids, not much seems to be known about their exact forms for one-dimensional chains. Even for the simple harmonic chain only few articles exist $25|26| 27 \mid 28$. 


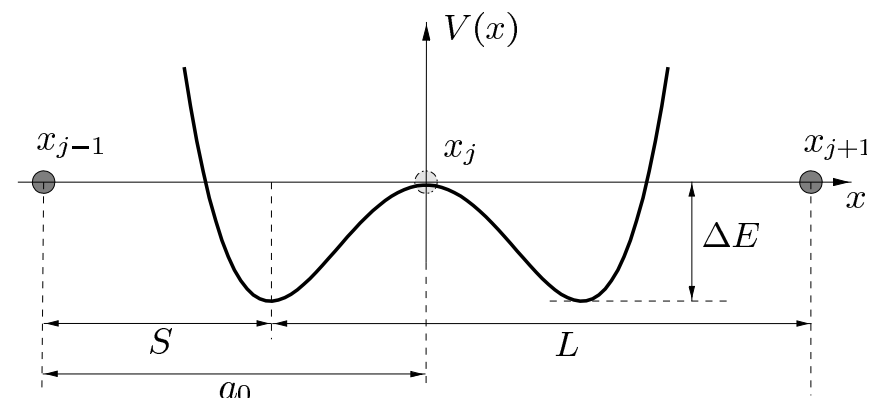

FIG. 1: Double-well potential $V(x)=x^{4}-2 x^{2}$ of the dynamic Fibonacci chain. The equilibrium distances are $S$ and $L$.

\section{B. Model systems}

As a simple one-dimensional model for a quasiperiodic system we consider the Fibonacci chain. It consists of particles arranged at two different distances: large ones $(L)$ and small ones $(S)$. The length ratio $L / S$ equals the number of the golden mean $\tau=\frac{1}{2}(\sqrt{5}+1)$. The sequence of the distances is created recursively by the mapping $\{L, S\} \mapsto\{L S, L\}$ with starting condition $L$. For example, after four iterations the resulting sequence is $L S L L S L S L$.

We want to study chains consisting of identical particles with nearest-neighbor interactions. The Hamiltonian has the form

$$
\mathcal{H}=\sum_{j=1}^{N} \frac{p_{j}^{2}}{2}+V\left(x_{j}-x_{j+1}-a_{j}\right),
$$

where $x_{j}$ and $p_{j}$ are position and momentum of the $j$ th particle. The dynamic Fibonacci chain (DFC) is defined by the choices $a_{j} \equiv a_{0}=\tau^{3}$ for the equilibrium distances and $V(x)=x^{4}-2 x^{2}$ for the interaction potential, respectively. The latter forms a double-well potential with minima at \pm 1 and a potential hill of height $\Delta E=1$ as shown in Fig. 1. Because neighboring particles sit in either of the potential minima, two nearest-neighbor distances $L=a_{0}+1$ and $S=a_{0}-1$ are possible. They fulfil the constraint $L / S=\tau$ of the Fibonacci chain.

The DFC shows two types of elementary excitations: Phonon vibrations in the local minima and phason flips that interchange the particle distances $L$ and $S$. At low temperatures only phonons are excited, phason flips have to be activated thermally. With its neighbors at rest the activation energy of a particle for a phason flip is $2 \Delta E$. This value is a result of the perfect superposition of the potential hills of both neigbors. The value is lowered when the neighbors assist by stepping simultaneously to the inside or outside during the phason flip thus creating a non-perfect superposition of the potential hills. Since the particle distances $L$ and $S$ are energetically degenerate, the total equilibrium potential energy is invariant under a phason flip.

The occurrence of phason flips makes nonlinearity an intrinsic feature of the DFC and an analytical treatment of the dynamics impossible. To understand the influence of the nonlinearity, we study four model systems with increasing complexity concerning their dynamical behavior:

- Harmonic periodic chain (HPC): $V_{\mathrm{HPC}}(x)=4 x^{2}$ and $a_{j}=a=2 \sqrt{5}$.

- Harmonic Fibonacci chain (HFC): $V_{\mathrm{HFC}}(x)=4 x^{2}$ and $a_{j}=L$ or $S$ according to the Fibonacci sequence.

- Dynamic Fibonacci chain (DFC): $V_{\mathrm{DFC}}(x)=x^{4}-2 x^{2}$ and $a_{j}=\tau^{3}$.

- Asymmetric Fibonacci chain (AFC): $V_{\mathrm{AFC}}(x)=V_{\mathrm{DFC}}+\chi\left(x^{2}-1\right)^{2}\left(\epsilon x+x^{2} / 2-1 / 2\right)$ and $a_{j}=\tau^{3}$ with $\chi \in[0,1]$ and $\epsilon= \pm 1$.

The potentials of the HPC, HFC, and DFC are chosen to be identical in the harmonic approximation around the equilibrium separation. The average particle distance $a$ is the same for all four systems. In the case of the Fibonacci chain the occurrence probabilities for $\mathrm{L}$ and $\mathrm{S}$ are given by $\tau^{-1}$ and $\tau^{-2}$, hence $a=2 \sqrt{5}$.

For the solution of the equations of motions we use a special molecular dynamics (MD) code. The code is introduced in Sec. II together with a short theoretical background. The simplest system is, of course, the HPC. Exact solutions for the equation of motion exist as a superposition of plane waves. We study the dynamics of the HPC in Sec. III as a reference system. The HFC consists of particles arranged on the Fibonacci chain with distances $L$ and $S$ interacting with the same harmonic potentials as the HPC, see Sec. IV. The DFC will then be studied in Sec. $\mathrm{V}$. In the case of the AFC the particles in the two potential minima have different eigenfrequencies. The parameters $\chi$ and $\epsilon$ determine the degree of asymmetry. For more details we refer to Sec. VI. We finish with a discussion and conclusion in Sec. VII.

\section{STRUCTURE FACTORS FROM MOLECULAR DYNAMICS}

\section{A. Definition of the structure factors}

We write the particle number density of the chain with $N$ particles as a sum of delta functions positioned along the particle trajectories $x_{l}(t), n(x, t)=\sum_{l=1}^{N} \delta\left(x-x_{l}(t)\right)$. The time dependent density-density correlation function and the density-density autocorrelation function are defined as

$$
\begin{aligned}
G(x, t) & =\frac{1}{N} \int\left\langle n\left(x^{\prime}, t\right) n\left(x+x^{\prime}, 0\right)\right\rangle \mathrm{d} x^{\prime} \\
& =\frac{1}{N} \sum_{j, l}\left\langle\delta\left(x-x_{j}(t)+x_{l}(0)\right)\right\rangle, \\
G_{\mathrm{a}}(x, t) & =\frac{1}{N} \sum_{l}\left\langle\delta\left(x-x_{l}(t)+x_{l}(0)\right)\right\rangle,
\end{aligned}
$$


where the brackets denote the thermal average. ${ }^{29}$ The coherent and incoherent structure factor are the spacetime Fourier transforms 30 ,

$$
\begin{aligned}
S(q, \omega) & =\frac{1}{2 \pi N} \int e^{-i \omega t} \sum_{j, l}\left\langle e^{-i q x_{j}(t)} e^{i q x_{l}(0)}\right\rangle \mathrm{d} t \\
S_{\mathrm{i}}(q, \omega) & =\frac{1}{2 \pi N} \int e^{-i \omega t} \sum_{l}\left\langle e^{-i q x_{l}(t)} e^{i q x_{l}(0)}\right\rangle \mathrm{d} t .
\end{aligned}
$$

Both functions are symmetric about $q=0$ and $\omega=0$. The static structure factor is the integral of the coherent structure factor, $S(q)=\int S(q, \omega) \mathrm{d} \omega$, i.e. the Fourier transform of $G(x, 0)$,

$$
S(q)=\frac{1}{N} \sum_{j, l}\left\langle e^{-i q x_{j}(0)} e^{i q x_{l}(0)}\right\rangle .
$$

\section{B. Molecular dynamics simulations}

For further calculations the particle trajectories are required as solutions of the equations of motion. Since in the case of the anharmonic chains only numerical solutions exist, we use a simple MD code. Initially the particles are placed on the equilibrium positions of a finite chain of length $L$ with periodic boundary conditions. The velocities are initialized according to a Gaussian distribution. Its width determines the total energy and thus the temperature of the system. The equations of motion are integrated by a Verlet algorithm running for a simulation time $T_{\mathrm{sim}}$. After starting the simulation, the dynamics is not controlled by a thermostat or in any other way.

For the direct numerical calculation of the Eqs. (3) we must compute a fourfold sum: two sums over the particle number $N$ and two over the time $T_{\text {sim }}$, one sum for the Fourier transform and one for the time average. Note, that by assuming ergodicity the thermal average \langle\rangle can be replaced by a time average $\frac{1}{T_{\text {sim }}} \int \mathrm{d} t$ and additionally by an average over several independent MD runs. For the sake of clarity the averaging over the MD runs is suppressed in the following notation. We introduce a more compact notation by defining the functions $f_{l}(q, t)=e^{i q x_{l}(t)}$. Let us assume tentatively that these functions are periodic in time with period $T_{\text {sim }}$ and in space with period $L$. Then the Eqs. (3) and (4) are greatly simplified to

$$
\begin{aligned}
S(q, \omega) & =\frac{1}{2 \pi N T_{\text {sim }}}\left\|\int e^{-i \omega t} \sum_{l} f_{l}(q, t) \mathrm{d} t\right\|^{2}, \\
S_{\mathrm{i}}(q, \omega) & =\frac{1}{2 \pi N T_{\text {sim }}} \sum_{l}\left\|\int e^{-i \omega t} f_{l}(q, t) \mathrm{d} t\right\|^{2}
\end{aligned}
$$

and

$$
S(q)=\frac{1}{N T_{\text {sim }}} \int\left\|\sum_{l} f_{l}(q, t)\right\|^{2} \mathrm{~d} t .
$$

The equations differ in the order of the absolute square and the particle sum. Since only two sums are left, an efficient numerical computation of the structure factors is possible. Furthermore a fast Fourier transform is used for the time integrals in Eqs. (5).

It is left to discuss the periodicity conditions. The spatial periodicity follows from the periodic boundaries used in the simulation. Therefore the chain acts like a ring and the excitations can go round during the simulation. To avoid such a behavior that would lead to unwanted correlations, we limit the maximum simulation time by the quotient of the length of the chain and the sound velocity $c_{s}$ to $T_{\max }=L / c_{\mathrm{s}}$. For all the model systems HPC, HFC, and DFC the sound velocity is the same, $c_{s}=a \sqrt{8}$, and $T_{\max }=N / \sqrt{8}$. We use $T_{\text {sim }}=T_{\max }$.

The functions $f_{l}(q, t)$ are in general not periodic in time. There is no reason, why the particles should be at the same positions at the end of the simulation as at the beginning. To avoid this problem, we multiply $f_{l}(q, t)$ with a window function $w(t)$ to enforce an artificial periodicity. The function $w(t)$ has to decrease fast enough - both in direct as in Fourier space - towards the boundaries of its domains. We use a normalized broad Gaussian function. Its width is chosen as large as possible with the constraint that the Gaussian has decayed to a small enough value at the interval boundaries. The effect of the Gaussian is a smoothing of the structure factors by convolution with a narrow Gaussian. The exact value of the width has no influence on the results.

\section{HARMONIC PERIODIC CHAIN AS REFERENCE SYSTEM}

\section{A. Analytic calculations}

The harmonic periodic chain (HPC) is used as a reference system to test our algorithms since its equations of motions can be solved analytically. If we put $x_{l}(t)=u_{l}(t)+l a$, then the $u_{l}(t)$ are expressed by a linear combination of normal modes. The wave vector $q$ and the frequency $\omega$ are related according to the dispersion relation $\omega(q)=2 \omega_{0}|\sin (q a / 2)|$. Here, $w_{0}$ is the eigenfrequency of a single particle. In the case of the model systems HPC, HFC, and DFC we have $\omega_{0}=\sqrt{8}$.

For the HPC the thermal averages in the structure factors, Eqs. (3) can be calculated to be

$$
\begin{aligned}
S(q, \omega) & =\frac{1}{2 \pi} \int e^{-i \omega t} \sum_{l=-\infty}^{\infty} e^{-i q a l} \exp \left(-\frac{1}{2} q^{2} \sigma_{l}^{2}(t)\right) \mathrm{d} t, \\
S_{\mathrm{i}}(q, \omega) & =\frac{1}{2 \pi} \int e^{-i \omega t} \exp \left(-\frac{1}{2} q^{2} \sigma_{0}^{2}(t)\right) \mathrm{d} t .
\end{aligned}
$$


where we used from the literature ${ }^{25}$

$$
\begin{aligned}
\sigma_{l}^{2}(t) & =\left\langle\left[u_{l}(t)-u_{0}(0)\right]^{2}\right\rangle \\
& =\frac{k_{B} T}{\omega_{0}^{2}}\left[l+\frac{1}{2} \int_{0}^{2 \omega_{0} t} J_{2 l}(s)\left(2 \omega_{0} t-s\right) \mathrm{d} s\right] .
\end{aligned}
$$

$J_{n}(s)$ is the Bessel function of the first kind of order $n$. The only external parameter in these functions is the temperature $T$. The particle sum and the Fourier transform have to be evaluated numerically. Due to the translation invariance of the HPC the double sum of the Eqs. (3) is reduced to a single sum. The static structure factor of the HPC can also be calculated analytically $\underline{26}$

$$
S(q)=\frac{\sinh \left(q^{2} \sigma^{2} / 2\right)}{\cosh \left(q^{2} \sigma^{2} / 2\right)-\cos (q a)},
$$

where $\sigma^{2}=k_{B} T / \omega_{0}^{2}$.

Let us take a closer look at the incoherent structure factor $S_{\mathrm{i}}(q, \omega)$. In the limit of small $T$ the term $\exp \left(-\frac{1}{2} q^{2} \sigma_{0}^{2}(t)\right)$ decays slowly with $t$ and we substitute $\sigma_{0}(t)$ with its approximation for large $t: \sigma_{0}(t)=$ $|t| k_{B} T / \omega_{0}$ for $|t| \rightarrow \infty$. This leads to a Lorentzian peak

$$
S_{\mathrm{i}}(q, \omega)=\frac{1}{\pi} \frac{\Gamma}{\Gamma^{2}+\omega^{2}}, \quad \Gamma=\frac{q^{2} k_{B} T}{2 \omega_{0}}
$$

In the limit of large $T$ the term $\sigma_{0}(t)$ is approximated for small $t: \sigma_{0}(t)=t^{2} k_{B} T$ for $|t| \ll \omega_{0}^{-1}$. Hence there is a Gaussian peak

$$
S_{\mathrm{i}}(q, \omega)=\frac{1}{\gamma \sqrt{2 \pi}} \exp \left(-\frac{\omega^{2}}{2 \gamma^{2}}\right), \quad \gamma=\sqrt{q^{2} k_{B} T} .
$$

The transition temperature between these two limiting cases is $k_{B} T=4 \omega_{0}^{2} / q^{2}$.

The Fourier transform of the Eqs. (7) yields the correlation functions

$$
\begin{aligned}
G(x, t) & =\frac{1}{\sqrt{2 \pi}} \sum_{l=-\infty}^{\infty} \frac{1}{\sigma_{l}(t)} \exp \left(-\frac{(x+l a)^{2}}{2 \sigma_{l}^{2}(t)}\right), \\
G_{\mathrm{a}}(x, t) & =\frac{1}{\sqrt{2 \pi}} \frac{1}{\sigma_{0}(t)} \exp \left(-\frac{x^{2}}{2 \sigma_{0}^{2}(t)}\right) .
\end{aligned}
$$

The function $G(x, t)$ is shown in Fig. 2. It consists of a sum of Gaussians centered at the equilibrium positions of the particles. The width of the Gaussians increases with temperature, as well as with time and in space: $\lim _{x, t \rightarrow \infty} G(x, t)=\rho$ and $\rho=1 / a$. This means that there is no longe-range order. Indeed, for the particle number density we have $\langle n(x)\rangle=\rho$, which is uniform as in liquids ${ }^{25}$. The autocorrelation function $G_{\mathrm{a}}(x, t)$ corresponds to the center peak at $x=0$.

\section{B. Simulation results}

In the case of the incoherent structure factor the variables $T$ and $q$ only appear as combination $q^{2} k_{B} T$ in

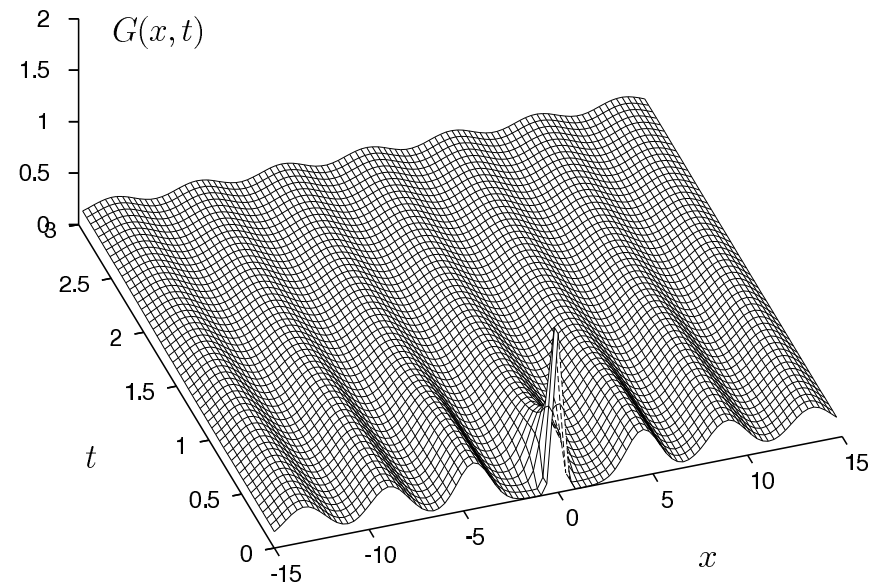

FIG. 2: Density-density correlation function $G(x, t)$ of the HPC for $k_{B} T=0.5$. The Gaussians are centered at integer multiples of $x=a \approx 4.47$.

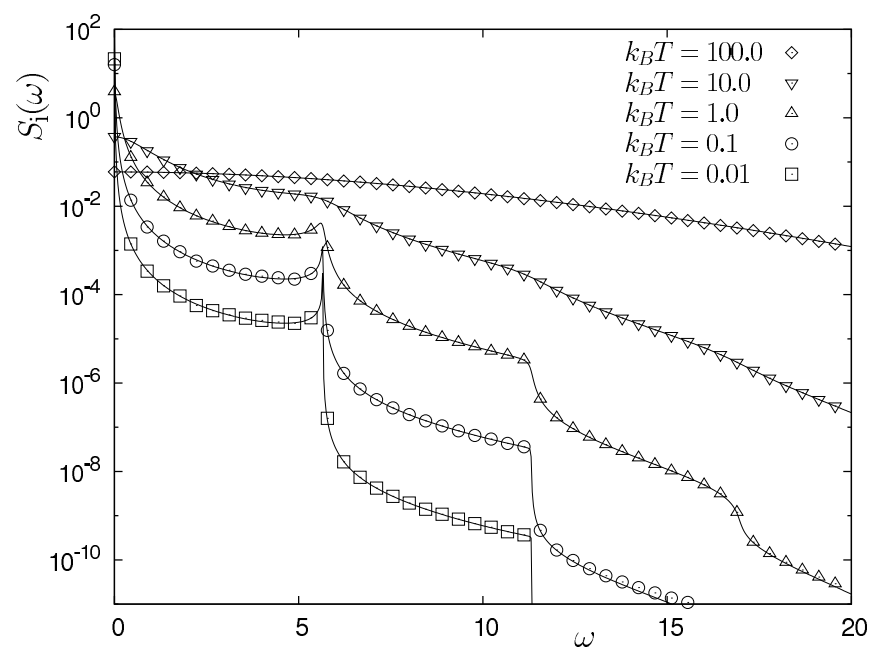

FIG. 3: Incoherent structure factor $S_{\mathrm{i}}(q, \omega)$ of the HPC for $q=\pi / a$, and different temperatures. The symbols mark the data from MD simulations with $N=1000$ particles, the lines the result from the analytical fomula, Eq. 7b . The peaks and edges are at integer multiples of $\omega=2 \omega_{0}=2 \sqrt{8}$.

Eqs. (7) and (8). Therefore it suffices to examine $S_{\mathrm{i}}$ at a fixed wave vector for different temperatures. We choose $q=\pi / a$ arbitrarily. The results from MD simulation and the numerical integration of the analytical formula, Eq. (7b) are shown in Fig. 3 for temperatures ranging from 0.01 to 100.0 . There is a maximum at $\omega=0$, called the quasielastic peak. In the low temperature regime the maximum has a Lorentzian shape. Furthermore a onephonon peak, a two-phonon edge, a three-phonon edge, etc. are found at $2 \omega_{0} \approx 5.7,4 \omega_{0} \approx 11.3,6 \omega_{0} \approx 17.0 .31$ Note that the multi-phonon contributions rapidly decay for larger $\omega$ (logarithmic scale). At higher temperatures the curve smoothes and approaches a Gaussian profile.

The coherent structure factor $S(q, \omega)$ is shown in Fig. 4. For a low temperature of $k_{B} T=0.01$ a one-phonon 
branch, a two-phonon branch, and very weakly a threephonon branch are observed. The one-phonon branch has a Lorentzian line shape and follows the phonondispersion relation. At higher $q$-values the branch broadens with a width proportional to $k_{B} T q^{2}$. This is similar to the temperature behavior for the incoherent structure factor. The multi-phonon branches follow the modified relations $\omega(q)=2 n \omega_{0}|\sin (q a / 2 n)|$ with $n=2,3$.

In Fig. 4(b) the comparison of the MD simulation (left side) and the analytical formula Eq. 7ap (right side) is shown. The temperature in this figure is $k_{B} T=0.1$, which is higher than in Fig. 4(a). As a consequence the one-phonon branch is broader. For both methods of calculating $S(q, \omega)$ a high accuracy over 12 orders of magnitude is possible. The accuracy is only limited by the internal floating point precision.

The static structure factor from Eq. (9) is compared to the results from the MD simulation in Fig. 5. $S(q)$ consists of a sequence of Lorentzian peaks at the reciprocal lattice points. The increasing width for larger wave vectors shows again that no long-range order is present in the one-dimensional model system.

The MD simulations and analytical formulas show a perfect agreement. Therefore we conclude that MD simulations are a well-suited numerical tool for calculating the structure factors of the one-dimensional model systems. Although we integrate the equations of motions with a good precision only on a short time scale using the simple Verlet-algorithm, the statistics extracted from the trajectories are correct. This confirms our approach and encourages us to proceed studying the phason dynamics of Fibonacci chains in the next section.

\section{HARMONIC FIBONACCI CHAIN: INFLUENCE OF THE QUASIPERIODICITY}

By changing the interparticle equilibrium distances of the HPC to those of a Fibonacci sequence with separations $L$ and $S$ we obtain the harmonic Fibonacci chain (HFC). The interaction potential is left unchanged. Since the incoherent structure factor is a function of the single particle motion only, it does not depend on the equilibrium distances of the particles but only the interaction potential. Hence, the incoherent structure factor of the HPC and of the HFC are identical. For the coherent structure factor the interparticle distances become important. Instead of Eq. (7a) we now have

$$
S(q, \omega)=\frac{1}{2 \pi} \int e^{-i \omega t} \sum_{l} e^{-i q x_{l}^{0}} \exp \left(-q^{2} \sigma_{l}^{2}(t) / 2\right) \mathrm{d} t .
$$

Here $x_{l}^{0}=\sum_{j=1}^{l} a_{j}$ for $l>0, x_{l}^{0}=\sum_{j=l}^{-1} a_{j}$ for $l<0$ and $x_{0}^{0}=0$ denote the equilibrium positions of the particles. For $k_{B} T=0$ this gives the Fourier transform of the static Fibonacci chain

$$
S(q, \omega)=\delta(\omega) \sum_{l} e^{-i q x_{l}^{0}}
$$

which is well known ${ }^{32}$. It consists of a dense set of delta peaks with varying intensity, positioned at the reciprocal lattice points

$$
q=q_{0}\left(h+\tau h^{\prime}\right), \quad h, h^{\prime} \in \mathbb{N}
$$

with $q_{0}=\frac{2 \pi}{a} \approx 1.40$.

As shown in Fig. 6 for the temperature $k_{B} T=0.02$, the coherent structure factor $S(q, \omega)$ of the HFC consists of many different branches all following the one-phonon dispersion relation. Relative to each other the branches are displaced in the $q$ direction. They start at the reciprocal lattice points with the intensity of the respective delta peak. Two-phonon branches are also found. The broadening of the branches proportional to $k_{B} T q^{2}$ has already been discussed for the HPC.

From these findings and Fig. 5 one can guess the form of the static structure factor $S(q)$ for the HFC: Lorentzians are positioned at the reciprocal lattice points with varying intensity. In Fig. $7 . S(q)$ is shown for $q \in[0,3 \pi]$. For small $q$ a large number of Lorentzians occur. With increasing $q$-value their width increases and the stronger ones hide the weaker ones. Comparing the position of the strong peaks and ignoring the change of their widths and heights, there is a self-similarity in $S(q)$. The deflation factor is $\Delta q_{1} / \Delta q_{2}=\tau^{3}$ as indicated in Fig. 7.

It is interesting to note that there are regions with very few peaks. They are positioned around $q=\pi, q=2 \pi$, etc. . The same regions are also special for the coherent structure factor. As seen in Fig. 6 all the weak onephonon branches vanish towards $q=\pi$. Only the strong one-phonon branch starting from the Bragg peak at $q=$ $\tau q_{0} \approx 2.27$ remains.

\section{DYNAMIC FIBONACCI CHAIN: OCCURRENCE OF PHASON FLIPS}

\section{A. Phason flips}

Let us now proceed to the anharmonic chains with phason flips by investigating the dynamic Fibonacci chain (DFC). It is built from identical particles that interact with a symmetric double-well potential $V_{\mathrm{DFC}}=x^{4}-2 x^{2}$. First the notion of a phason flip has to be specified. To do so we identify the position of the changes from $L$ to $S$ and from $S$ to $L$ of the interparticle distances along the particle trajectories. This is done in Fig. 8. Often a $L \rightarrow S$ change and a $S \rightarrow L$ change lie next to each other (particle distance 1 ) and the sequences $L S$ and $S L$ are interchanged. But there are also many cases where the positions of the two changes are separated by 0,2 , 3 , or even more particle distances as marked by lines in Fig. 8. Sometimes it is not possible to find a partner locally. Only in the long time average every $L \rightarrow S$ change will eventually cancel with a $S \rightarrow L$ change.

In the literature on the Fibonacci chain a phason flip is understood as the exchange of a $L$ and a neighboring 

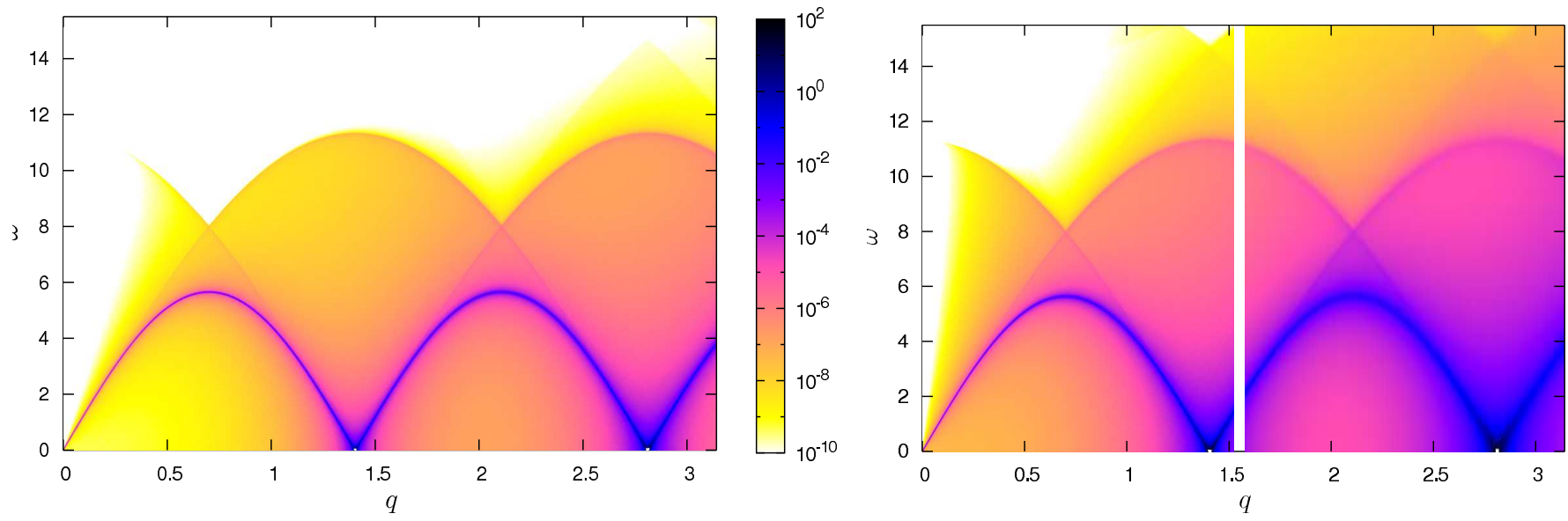

FIG. 4: (Color online) Coherent structure factor $S(q, \omega)$ of the HPC with $N=6500$ particles from MD simulation. The temperatures are for $k_{B} T=0.01$ (a) and for $k_{B} T=0.1$ (b). One-, two- and three-phonon branches are observed. They start at the reciprocal lattice points $2 \pi n / a$. In (b) the output from the MD simulation (left side) is compared to the output of the analytical formula Eq. 7a) (right side).

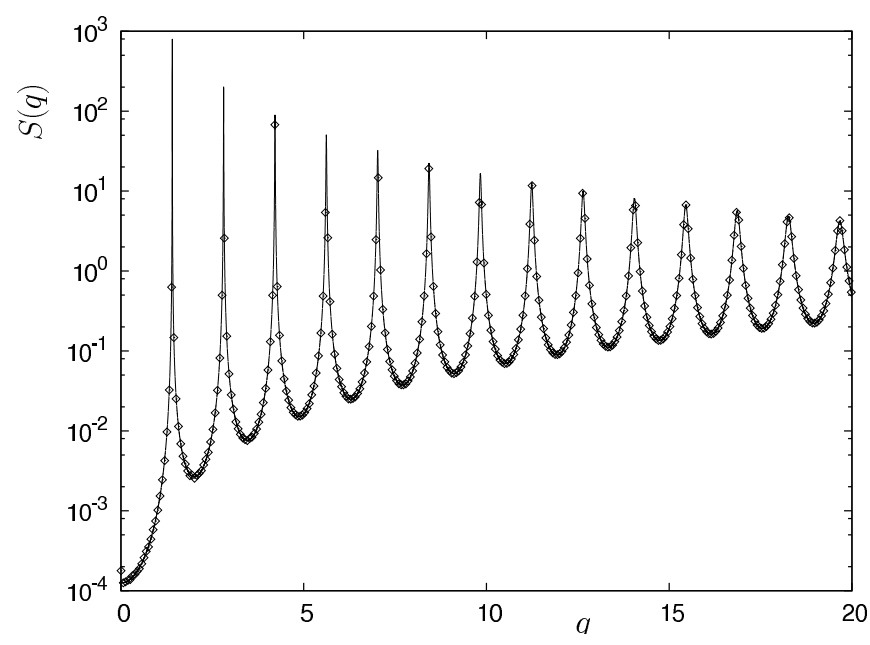

FIG. 5: Static structure factor $S(q)$ of the HPC for $k_{B} T=$ 0.02. The symbols mark the data from a MD simulation with $N=1000$ particles, the line the result from the analytical fomula, Eq. (9).

$S$ particle distance. As we learned above there are also other types of exchanges in the DFC. In the following we denote by phason flip every pair of flip partners as those connected by lines in Fig. 8. Note that the times and positions of the phason flips are not well defined. Only their number can be estimated by counting the changes in the particle distances as we will do now.

The temperature dependence of the average phason flip frequency $\omega_{\text {flip }}$ is shown in Fig. 9 Phason flips start to appear at about $k_{B} T=0.1$. At low $k_{B} T$ the average phason flip frequency increases rapidly by thermal excitation and $\omega_{\text {flip }} \ll \omega_{0}$. At higher temperatures $k_{B} T>0.4$ the average phason flip frequency slowly saturates. In this region the internal energy is comparable to the potential hill.

\section{B. Results for the structure factors}

For the anharmonic chains no analytic results are available, in particular not at elevated temperatures when phason flips occur. The incoherent structure factors of the DFC and of the HFC/HPC differ remarkably and are shown in Fig. 10 for $q=\pi / a$. The comparison leads to the following conclusions:

1. At a fixed temperature, there are $\omega$-ranges where the curve for the DFC lies below the curve for the HFC/HPC and vice versa. Since we have $\int S_{\mathrm{i}}(q, \omega) \mathrm{d} \omega=1$ from Eq. (3b), the integral area between the two curves has to vanish.

2. At very low $k_{B} T$ and $\omega<2 \omega_{0} \approx 5.7$ the curves of the DFC and the HFC/HPC cannot be distinguished in logarithmic scale except for two small bumps. They are a consequence of the anharmonicity of the interaction potential of the DFC and not related to the phason flips. At larger $\omega$ values the multi-phonon edges have different heights.

3. Above $k_{B} T=0.1$ the one-phonon peak and the multi-phonon edges in the curves for the DFC broaden and weaken considerably faster than in the curves for the HFC/HPC. They finally disappear at $k_{B} T=1.0$.

4. No additional peaks or edges occur at any temperature.

Further MD runs show that different $q$-values change the temperature dependence, but the general features remain unchanged: The phonon peaks and edges broaden and weaken much faster with increasing temperature for the DFC than for the HFC/HPC.

Similar conclusions follow for the coherent structure factor of the DFC. $S(q, \omega)$ for the DFC looks qualitatively 


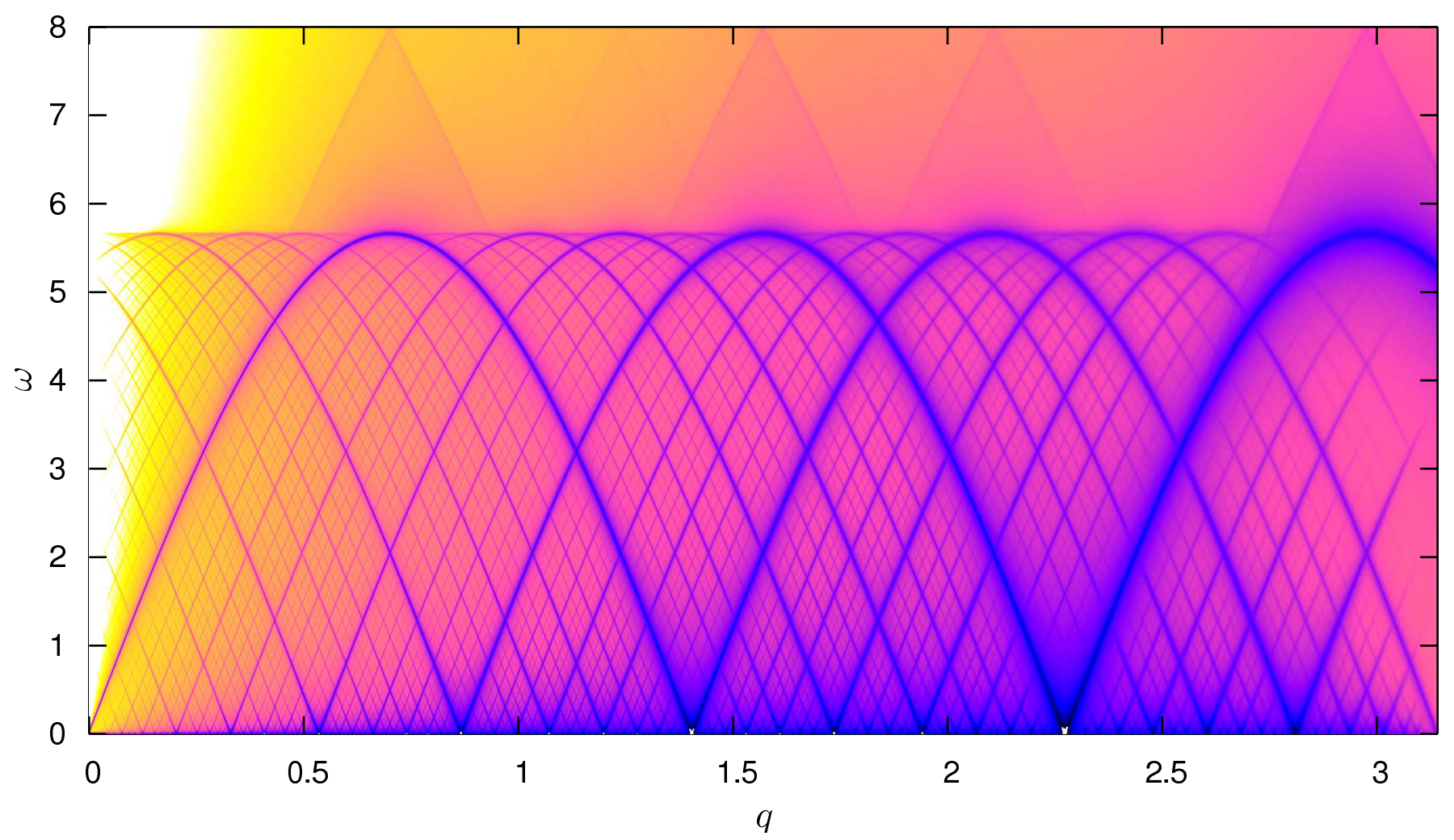

FIG. 6: (Color online) Coherent structure factor $S(q, \omega)$ of the HFC with $N=13000$ particles for $k_{B} T=0.02$. It consists of a dense set of phonon branches starting from the reciprocal lattice points.

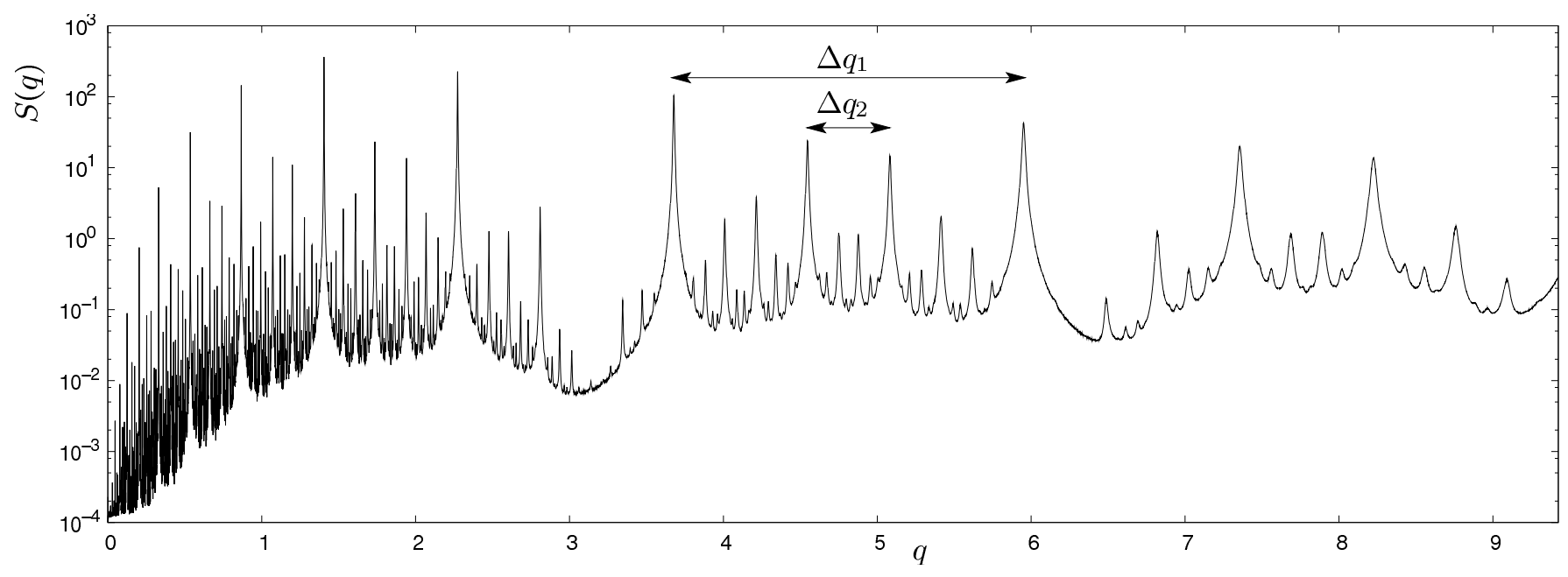

FIG. 7: Static structure factor $S(q)$ of the HFC from a MD simulation with 2000 particles at the temperature $k_{B} T=0.02$.

similar to $S(q, \omega)$ for the HFC except that the branches broaden more quickly with temperature. To compare the broadening let us look at plane cuts through $S(q, \omega)$ for $\omega=1.0$ fixed. Three cuts for the temperature $k_{B} T=$ $0.05,0.2$, and 0.3 are shown in Fig. 11. A one-phonon branch is located inside the cut resulting in a peak with approximate Lorentzian line shape as indicated by the fits in the figure. Only for lower temperatures the line shape deviates from a Lorentzian, which is seen at the base of the peak for $k_{B} T=0.05$.

The width of the Lorentzian in Fig. 11 is shown as a function of temperature in Fig. 12 for both, the HFC and the DFC. In the case of the HFC the width increases linearly with temperature, as discussed in Sec. IV] There is a deviation from linearity at low temperatures. This is an artefact from the method of calculation from MD simulation data. As explained in Sec. II B the structure factor is convoluted with a Gaussian due to the finite 


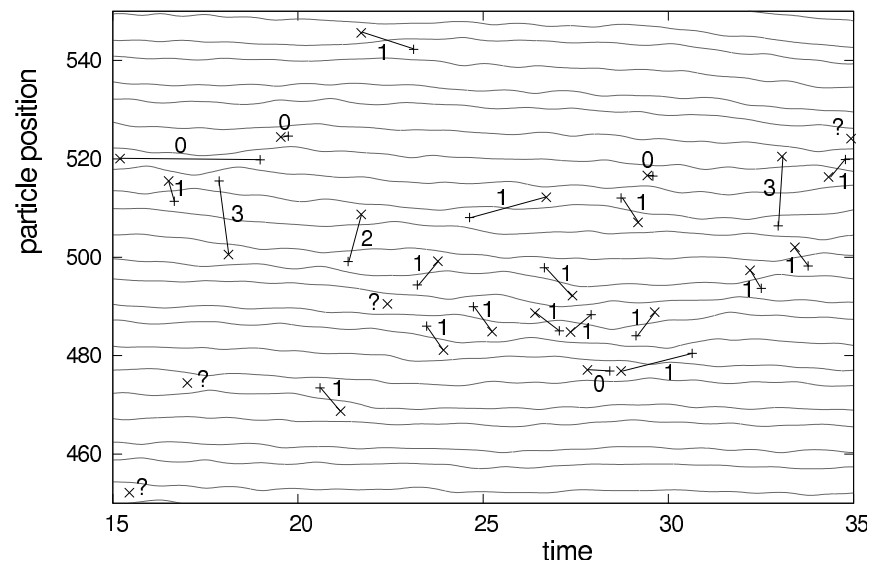

FIG. 8: Snapshot of the particle trajectories of the DFC at the temperature $k_{B} T=0.6$. Changes in the particle distances from $L$ to $S$ and $S$ to $L$ are marked with a cross $(\times)$ and a plus (+). A $L \rightarrow S$ change and a $S \rightarrow L$ change combined form a phason flip.

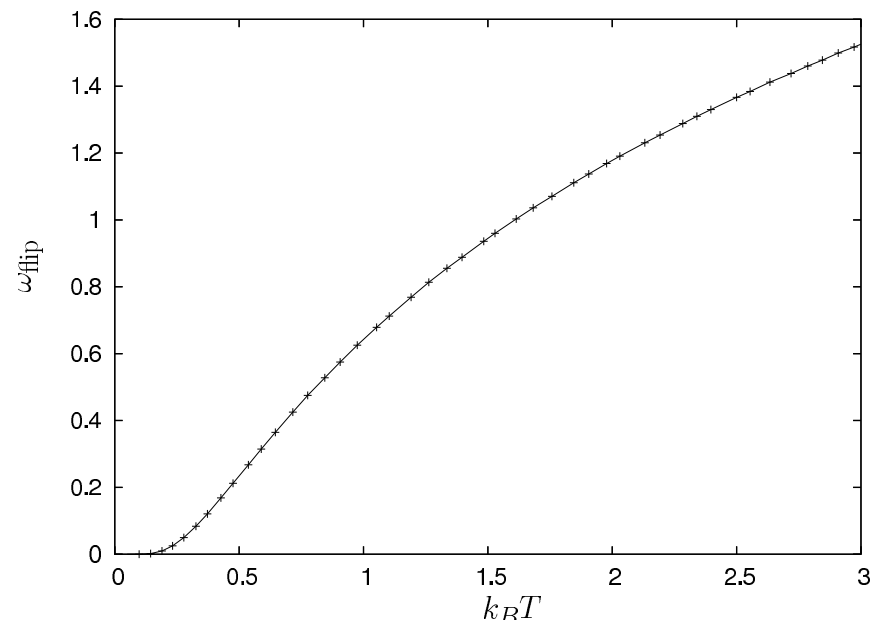

FIG. 9: Average flip frequency as a function of the temperature $k_{B} T$. In the temperature range of the figure: $\omega_{\text {flip }}<$ $\omega_{0} \approx 2.83$.

simulation time. The (narrow) Gaussian generates the observed offset. The convolution with the Gaussian is also responsible for the shape of the cuts in Fig. 11 at low temperature, deviating from the Lorentzian shape.

There is one aspect of the DFC that has been ignored up to now. Due to the phason flips the original perfect quasiperiodic long-range order is slowly decaying. With progressing simulation time the chain becomes randomized which, however, has no effect on the incoherent structure factor. To test the influence of the randomization on the coherent structure factor, a MD simulation was started with a totally randomized Fibonacci chain. The interaction potentials and the occurrence ratio of $L$ and $S$ were not adapted. The result of the simulation is shown in Fig. 13. Most of the details in $S(q, \omega)$ are lost by the randomization process and the branches are strongly

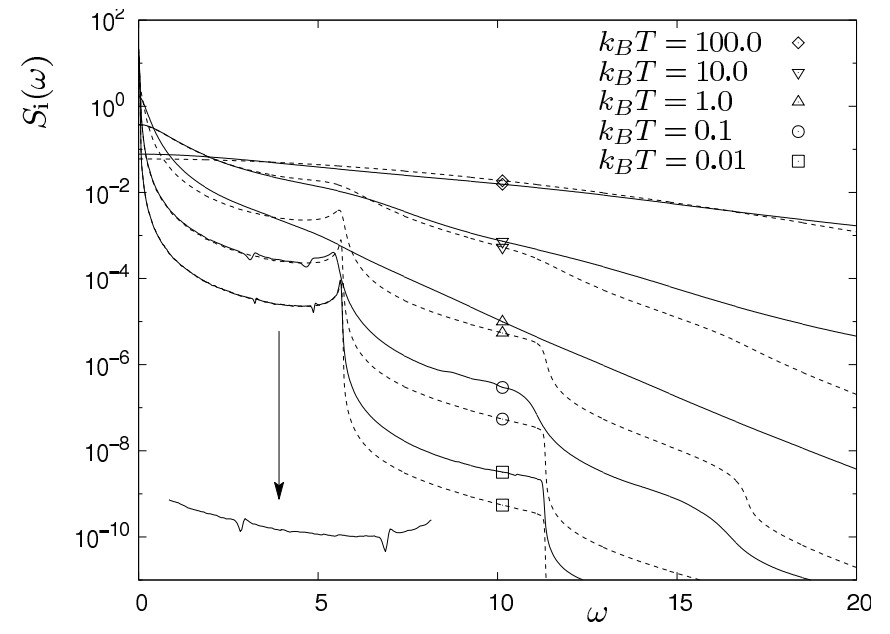

FIG. 10: Incoherent structure factor $S_{\mathrm{i}}(q, \omega)$ of the DFC (solid) and the HFC/HPC (dashed) for $q=\pi / a$ at different temperatures. The data was calculated using MD simulations with $N=1000$ particles.

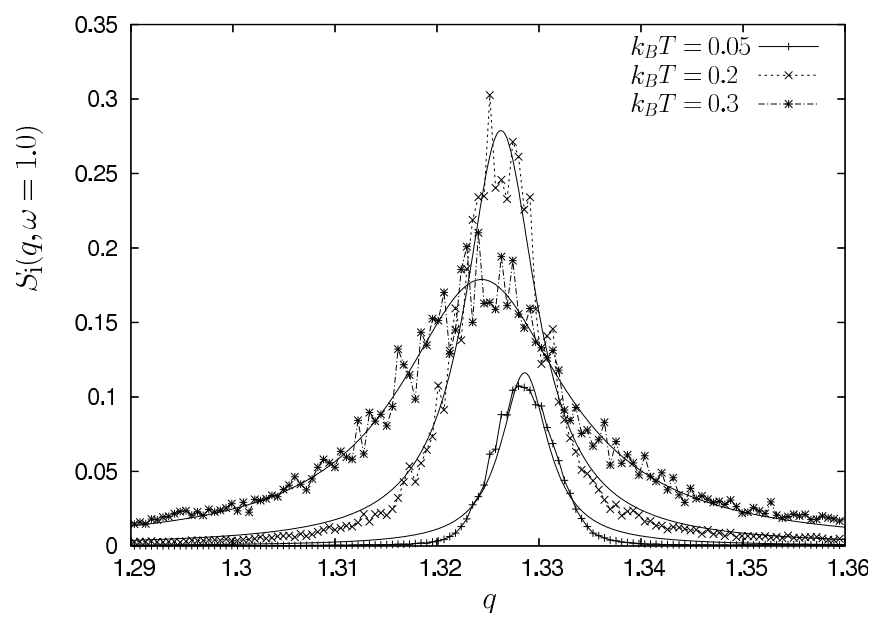

FIG. 11: Cuts through $S(q, \omega)$ of the DFC for a fixed $\omega=1.0$ including a one-phonon peak. The solid curves are fits with Lorentzians.

broadened, although the simulation has been carried out at a low temperature of $k_{B} T=0.02$.

We summarize the results of this section: By the introduction of the anharmonic double-well potential of the DFC the phonon peaks, edges, and branches are strongly broadened and weakened with increasing temperature. There are two effects responsible for the broadening: (1) The anharmonicity and single phason flips affect the propagation of phonons. This is seen in the incoherent structure factor. (2) The destruction of the long-range order by a large number of phason flips. This is the main effect of broadening for the coherent structure factor. The phason flips appear more or less uniformly distributed over the simulation time and the particles. 


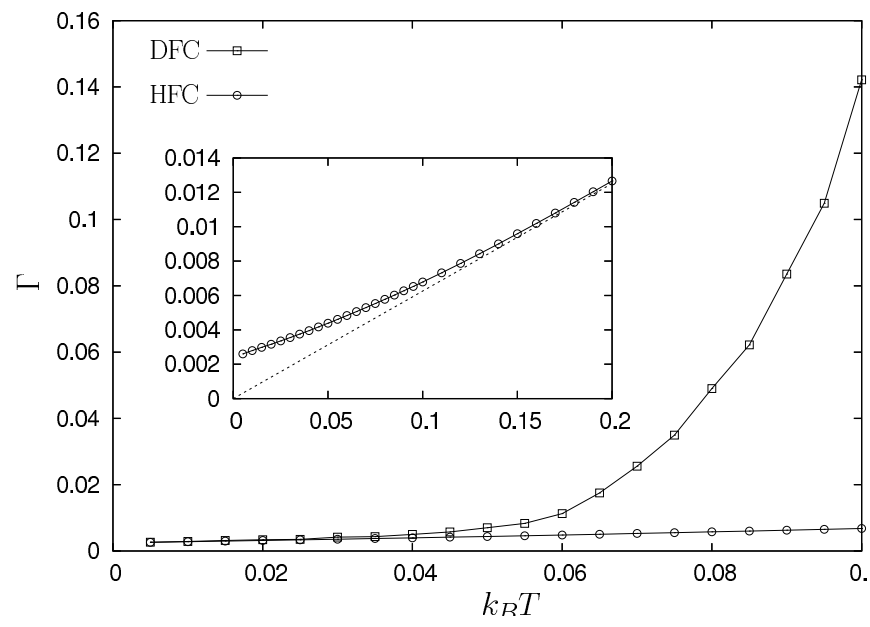

FIG. 12: Width of the Lorentzian peak of Fig. 11 as a function of temperature for the HFC (inset) and the DFC.

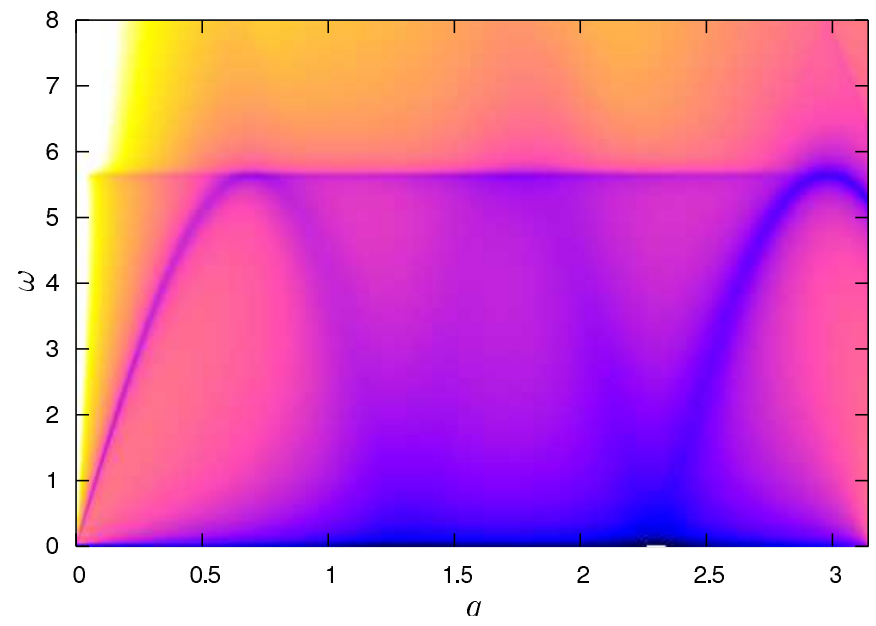

FIG. 13: (Color online) Coherent structure factor $S(q, \omega)$ of a randomized Fibonacci chain with 2000 particles at the temperature $k_{B} T=0.02$. The same interaction potentials as in Fig. 6 have been used.

\section{ASYMMETRIC FIBONACCI CHAIN: COMPETING EIGENFREQUENCIES}

\section{A. Band gaps}

In the next step we modify the double well potential of the DFC. Like all the previous model systems the asymmetric Fibonacci chain (AFC) is built of identical particles, but they interact with the more complicated potential $V_{\mathrm{AFC}}=V_{\mathrm{DFC}}+\Delta V$, see Fig. 14. The additional term is given by

$$
\Delta V(x)=\chi\left(x^{2}-1\right)^{2}\left(\epsilon x+x^{2} / 2-1 / 2\right)
$$

with $\chi \in[0,1]$ and $\epsilon= \pm 1$. This term has been chosen in such a way that the positions of the minima at $x= \pm 1$ are left invariant, but the curvatures of the potential at those points is changed to $V_{\mathrm{AFC}}^{\prime \prime}( \pm 1)=8(1 \pm \epsilon \chi)$.
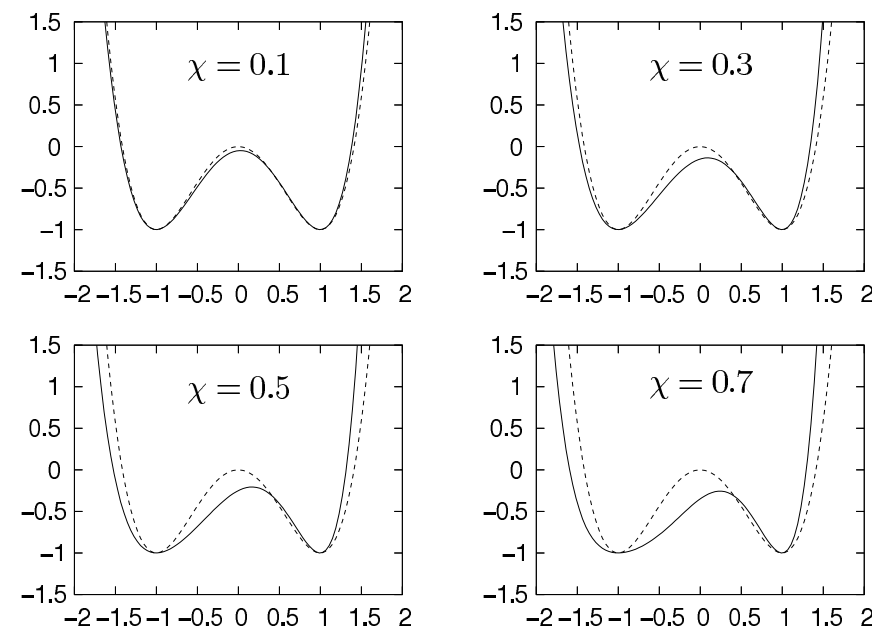

FIG. 14: Interaction potential $V_{\mathrm{AFC}}(x)$ of the $\mathrm{AFC}$ for different values of the parameter $\chi$ and $\epsilon=1$. $V_{\mathrm{DFC}}$ is shown with dashed lines.

In harmonic approximation a particle will feel different eigenfrequencies, depending on the nearest-neighbor configuration $S S, S L, L S$, or $L L$. In the case of $\epsilon=1$ it is $\omega_{\mathrm{LL}}>\omega_{\mathrm{LS}}=\omega_{\mathrm{SL}}>\omega_{\mathrm{SS}}$ and for $\epsilon=-1$ it is $\omega_{\mathrm{LL}}<\omega_{\mathrm{LS}}=\omega_{\mathrm{SL}}<\omega_{\mathrm{SS}}$. The sign change of $\epsilon$ corresponds to a mirror operation of $V_{\mathrm{AFC}}$ about the $y$-axis.

The coherent structure factor of the AFC with $\chi=0.1$, $0.3,0.5$ and $\epsilon= \pm 1$ is shown in Fig. 15. Band gaps of different widths appear and broaden with increasing values for $\chi$. They are positioned at the frequencies where one-phonon branches intersect each other. Their positions and widths are different for $\epsilon=1$ and $\epsilon=-1$. For $\epsilon=1$ three large gaps and several smaller gaps appear, whereas for $\epsilon=-1$ only one very large gap, one medium gap and several small gaps appear. The band gaps are a consequence of the competing eigenfrequencies due to the asymmetric potential in the same way as band gaps appear in periodic systems with several particles and eigenfrequencies per unit cell.

\section{B. Density of states}

The gaps are seen more clearly in the density of states (DOS) $D(\omega)$. The DOS per particle is calculated from the velocity autocorrelation function by a Fourier transform

$$
D(\omega)=\frac{1}{\pi N} \int e^{-i \omega t} \sum_{l}\left\langle v_{l}(t) v_{l}(0)\right\rangle \mathrm{d} t, \quad \omega \geq 0 .
$$

It is obtained from MD simulation data similar to the incoherent structure factor: $f_{l}(q, t)$ has to be substited by $v_{l}(t)$ in Eq. (5b). By interchanging the Fourier transform and two time derivatives we can alternatively write

$$
D(\omega)=\frac{\omega^{2}}{\pi N} \int e^{-i \omega t} \sum_{l}\left\langle x_{l}(t) x_{l}(0)\right\rangle \mathrm{d} t .
$$



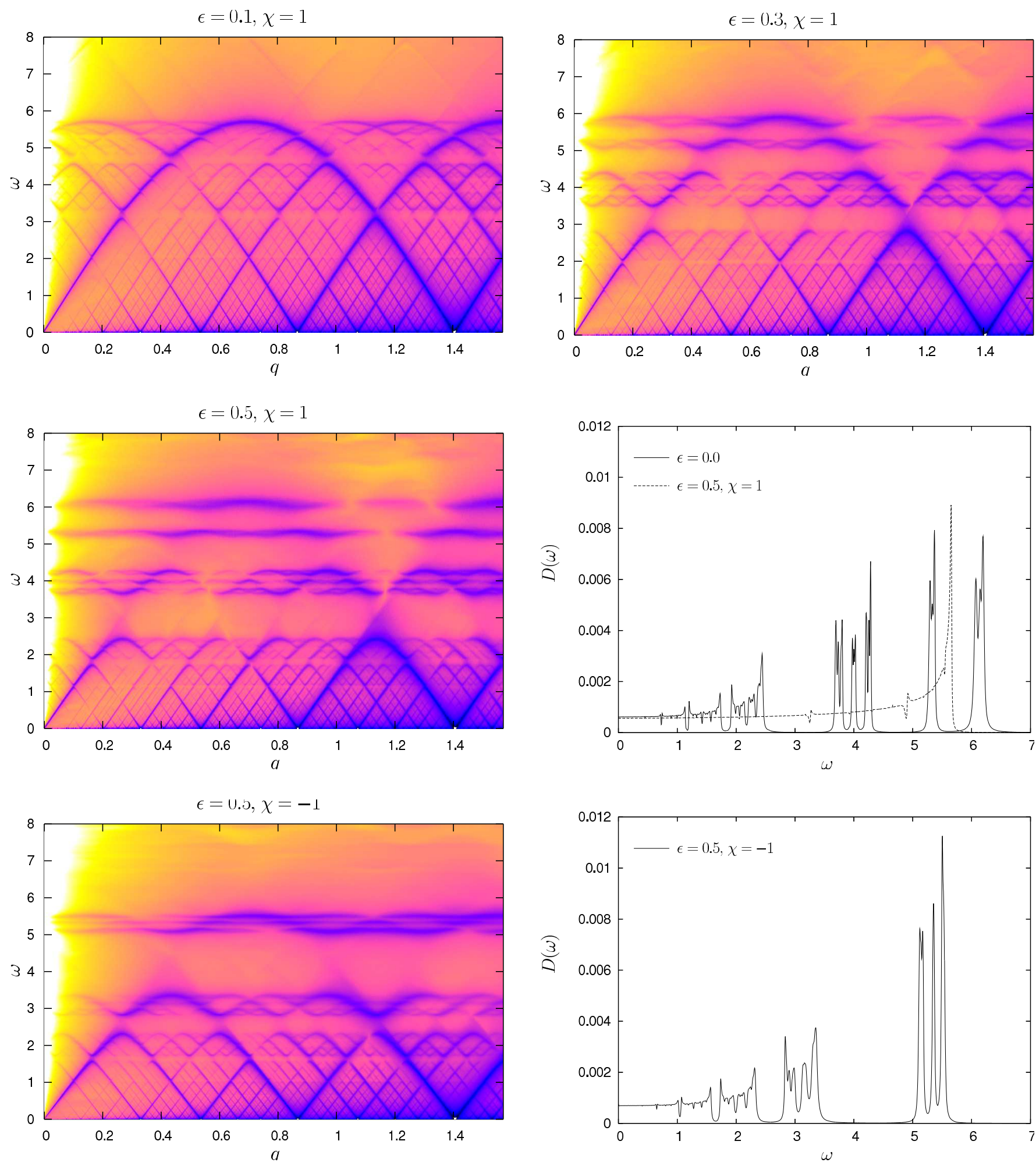

FIG. 15: (Color online) Coherent structure factor $S(q, \omega)$ of the AFC for different values of $\chi$ and $\epsilon$ from MD simulations with 6500 particles at $k_{B} T=0.01$. Band gaps are observed. The band gaps also appear in the DOS $D(\omega)$. 
After a taylor expansion of the exponentials in Eq. 3b a connection to the incoherent structure factor is found,

$$
D(\omega)=2 \omega^{2} \lim _{q \rightarrow 0} \frac{S_{\mathrm{i}}(q, \omega)}{q^{2}}, \quad \omega \neq 0 .
$$

For comparison we present the DOS of a harmonic chain (HPC or HFC)

$$
D(\omega)=\frac{2}{\pi} \frac{k_{B} T}{\sqrt{4 \omega_{0}^{2}-\omega^{2}}} \quad \text { for } \quad 0<\omega<2 \omega_{0}
$$

and 0 elsewhere. The total number of states is normalized to $\int_{0}^{\infty} D(\omega) \mathrm{d} \omega=k_{B} T$.

It can be checked, that the DOS of the harmonic chain fits well to the DOS of the AFC for $\epsilon=0.0$, except small bumps that originate from the anharmonicity of the potentials. In Fig. 15 the DOS of the AFC for different values of $\chi$ and $\epsilon$ are drawn. The band gaps appear at the same frequencies and the same widths as in the coherent structure factor.

\section{DISCUSSION AND CONCLUSION}

At the end we would like to make some general remarks concerning phason flips and phason modes: In the context of a hydrodynamic theory, phason flips can be associated with phason modes. It was noted quite early 33 , that phason modes are diffusive, in contrary to the propagating phonons. This means that phason flips are only weakly coherent in space and in time, which of course we have also observed here. As a result their influence on the structure factor is small making it difficult though still interesting to study them by scattering experiments. Another point concerns the connection of phason flips and quasiperiodicity. There is no reason why phason flips should only occur in quasicrystals. Since interaction potentials are not sensitive on the long-range order, phason flips in the form of atomic jumps can also occur in periodic complex intermetallic phases, which is supported by recent experimental results $34 \mid 35$. In the case of our model systems, it is equally possible to compare simulations of a periodic $L S L S L S \ldots$ chain with harmonic and double-well potentials respectively.

In conclusion, we have investigated the dynamics of phonons and phason flips in one-dimensional model systems with molecular dynamics simulations. An efficient algorithm made it possible to calculate the structure factors with high precision and in great detail. As a result multi-phonon contributions, - although weak in comparison to the one-phonon peaks and branches - have been identified. By introducing phasons in the model systems we were able to study their influence on the structure factors, which is mainly a broadening of the characteristic peaks, edges, and branches with temperature. The broadening can be further split into a broadening due to the disorder as a result of collective phason flips, i.e. a static phason field, and a broadening due to the anharmonicity of the interaction potentials and single phason flips. The work presented here is a first step. Further studies on two-dimensional and three-dimensional model systems with phason flips are under way.

\section{Acknowledgments}

We wish to thank T. Odagaki and M. Umezaki for stimulating discussions.
* Electronic address: mengel@itap.uni-stuttgart.de

1 An introduction on quasicrystals can be found in: C. Janot, Quasicrystals: A Primer (Oxford Science Publications, Oxford, 1992); M. de Boissieu, P, Guyot, and M. Audier, in Lectures on Quasicrystals, edited by D. G. F. Hippert (Les Éditions de Physique, Aussois, 1994), p. 1.

${ }^{2}$ For a review on phonon excitations in quasicrystals see M. Quilichini and T. Janssen, Rev. Mod. Phys. 69, 277 (1997) and references therein.

3 P. Bak, Phys. Rev. Lett. 54, 1517 (1985); P. A. Kalugin, A. Y. Kitayev, and L. S. Levitov, J. Physique Lett. 46, L-601 (1985); D. Levine, T. C. Lubensky, S. Ostlund, S. Ramaswamy, P. J. Steinhardt, J. Toner, Phys. Rev. Lett. 54, 1520 (1985).

4 M. de Boissieu, M. Boudard, B. Hennion, R. Bellissent, S. Kycia, A. Goldman, C. Janot, and M. Audier, Phys. Rev. Lett. 75, 89 (1995).

5 J. E. S. Socolar, T. C. Lubensky, and P. J. Steinhardt, Phys. Rev. B 34, 3345 (1986).

${ }^{6}$ R. Rosenfeld, M. Feuerbacher, B. Baufeld, M. Bartsch, M.
Wollgarten, G. Hanke, M. Beyss, U. Messerschmidt, K. Urban, Phil. Mag. Lett. 72, 375 (1995).

7 P. A. Kalugin and A. Katz, Europhys. Lett. 21, 921 (1993).

8 R. Bluher, P. Scharwaechter, W. Frank, and H. Kronmüller, Phys. Rev. Lett. 80, 1014 (1998).

${ }^{9}$ W. Steurer, Acta. Cryst. A 61, 28 (2005).

${ }^{10}$ K. Edagawa, K. Suzuki, S. Takeuchi, Phys. Rev. Lett 85, 1674 (2000).

11 E. Abe, S. J. Pennycook, and A. P. Tsai, Nature 421, 347 (2003).

12 M. Engel and H.-R. Trebin, Phil. Mag 85, 2227 (2005).

13 G. Coddens, S. Lyonnard, B. Hennion, Y. Calvayrac, Phys. Rev. B 62, 6268 (2000).

14 The coherent structure factor $S(q, \omega)$ is also called '(total) scattering function', 'response function', or 'dynamic(al) structure factor'. The incoherent structure factor $S_{\mathrm{i}}(q, \omega)$ is alternatively known as 'incoherent scattering function' and 'self part of the structure factor'.

15 M. de Boissieu, K. Shibata, R. Currat, A. Q. R. Baron, S. Tsutsui, A.-P. Tsai, J. Non-Cryst. Solids 334\&335, 303 
(2004) and references therein.

16 M. Krisch, R. A. Brand, M. Chernikov, H. R. Ott, Phys. Rev. B 65, 134201 (2002).

17 M. de Boissieu, M. Boudard, R. Bellissent, M. Quilichini, B. Hennion, R. Currat, A. I. Goldman, and C. Janot, J. Phys.: Condens. Matter 5, 4945 (1993).

18 M. Boudard, M. de Boissieu, S. Kycia, A. I. Goldman, B. Hennion, R. Bellissent, M. Quilichini, R. Currat, and C. Janot, J. Phys.: Condens. Matter 7, 7299 (1995).

19 S. Lyonnard, G. Coddens, Y. Calvayrac, and D. Gratias, Phys. Rev. B 53, 3150 (1996).

20 G. Coddens, S. Lyonnard, and Y. Calvayrac, Phys. Rev. Lett. 78, 4209 (1997).

21 G. Coddens, S. Lyonnard, B. Hennion, and Y. Calvayrac, Phys. Rev. Lett. 83, 3226 (1999).

22 J. A. Ashraff and R. B. Stinchcombe, Phys. Rev. B 39, 2670 (1989).

23 G. G. Naumis, C. Wang, M. F. Thorpe, and R. A. Barrio, Phys. Rev. B 59, 14302 (1999).

24 J. Hafner, M. Krajçí, J. Phys.: Condens. Matter 5, 2489 (1993).

25 T. Yoshida, K. Shobu, and H. Mori, Prog. Theor. Phys. 66, 759 (1981).

${ }^{26}$ V. J. Emery and J. D. Axe, Phys. Rev. Lett. 40, 1507 (1978).
27 J. Florencio and M. H. Lee, Phys. Rev. A 31, 3231 (1985).

28 G. Radons, J. Keller, and T. Geisel, Z. Phys. B 61, 339 (1985).

29 The correlation functions have the following interpretation: $G(x, t) \Delta x$ is the probability to find a particle in $[x, x+\Delta x]$ at time $t$, if any particle has been at the origin at $t=0$. $G_{\mathrm{a}}(x, t) \Delta x$ is the probability to find a particle in $[x, x+\Delta x]$ at time $t$, if the same particle has been at the origin at $t=0$.

30 M. Bée, Quasielastic neutron scattering, IOP Publishing, Bristol (1988).

31 The notation ' $\mathrm{x}$-phonon' is adopted freely from the language of neutron scattering. However in the case of a onedimensional system there is no regular phonon-expansion possible, as it is done for three-dimensional crystals.

32 D. Levine and P. J. Steinhardt, Phys. Rev. Lett. 53, 2477 (1984).

33 T. C. Lubensky, S. Ramaswamy, and J. Toner, Phys. Rev. B 32, 7444 (1985).

34 U. Dahlborg, W. S. Howells, M. Calvo-Dahlborg, and J. M. Dubois, J. Phys.: Condens. Matter 12, 4021 (2000).

35 J. Dolinšek, T. Apih, P. Jeglič, M. Klanjšek, J. Non-Cryst. Solids 334\&335, 280 (2004). 